\title{
A unique computational algorithm to simulate probabilistic multi-factor interaction model complex material point behavior
}

\author{
C. C. Chamis ${ }^{1}$ \& G. H. Abumeri ${ }^{2}$ \\ ${ }^{I}$ NASA Glenn Research Center, Cleveland, Ohio, USA \\ ${ }^{2}$ Alpha Star Corporation, Long Beach, California, USA
}

\begin{abstract}
The Multi-Factor Interaction Model (MFIM) is used to evaluate the divot weight (foam weight ejected) from the launch external tanks. The multi-factor has sufficient degrees of freedom to evaluate a large number of factors that may contribute to the divot ejection. It also accommodates all interactions by its product form. Each factor has an exponent that satisfies only two points - the initial and final points. The exponent describes a monotonic path from the initial condition to the final. The exponent values are selected so that the described path makes sense in the absence of experimental data. In the present investigation, the data used was obtained by testing simulated specimens in launching conditions. Results show that the MFIM is an effective method of describing the divot weight ejected under the conditions investigated.
\end{abstract}

Keywords: weight, comparisons, cumulative distribution, probabilistic sensitivities, local optimization.

\section{Introduction}

The simulation of complex material behavior resulting from the interaction of several factors (such as temperature, nonlinear material due to high stress, time dependence, fatigue, etc.) has been mainly performed by factor-specific representations. For example, entire text books are devoted to plasticity, creep, fatigue and high strain rate to mention only a few. Investigators have derived equations that describe material behavior for each factor-specific effect. Suppose we visualize that the material behavior is a continuum represented by some 
surface. Then, we can think of some representation which describes that surface which is inclusive of all participating factors that affect material behavior either singly or interactively in various combinations. To that end, research has been a continuing activity at NASA Glenn Research Center (GRC) for about thirty years. It started with a primitive form of the Multi-Factor Interaction Model (MFIM) representation for describing complex composite behavior in polymer matrix composites (Chamis et al. [1]). It was extended to metal matrix composites (Chamis and Hopkins [2]) and continued to be evolving during the National Aerospace Plane and the High Speed Research Programs (Chamis et al. [3]). The result of all this research is the development of the MFIM to represent complex material point behavior by a single equation (Tong et al. [4], Boyce and Chamis [5]). The development of this equation starts with the premise that, if we are to quantify the range of factors affecting material point properties, we need a description of point behavior (Minnetyan [6], Chamis and Minnetyan [7]). In this context, it is reasonable to consider that behavior constitutes an n-dimensional space (Point Behavior Space (PBS)) where each point on that surface represents a specific aspect of complex behavior. It is further reasonable to assume that PBS can be described by an assumed interpolation function. One convenient interpolation function is a polynomial of product form because mutual interactions among different factors can be represented by the overall product, and includes those cross products which are present in common algebraic polynomials.

\section{Multi-factor interaction model}

In this investigation, PBS is assumed to be described by the model shown in the following equation:

$$
\frac{P}{P_{6}}=\left[1-\frac{x_{1}}{x_{1 f}}\right]^{e x 1}\left[1-\frac{x_{2}}{x_{2 f}}\right]^{e x 2}\left[1-\frac{x_{3}}{x_{3 f}}\right]^{e x 3} \ldots\left[1-\frac{x_{n}}{x_{n f}}\right]^{e x n}
$$

where $\frac{P}{P_{6}}$ is the ratio of predicted effected property to some arbitrary original Property $\mathrm{P}_{\mathrm{o}} ; \frac{x_{i}}{x_{i f}}$ is the ratio factor (design variable that is known to influence the initial property) to some arbitrary final condition; ex 1 is an exponent which can be set to some default value (say 0.5 ), and $n$ is the total number of factors. The factor final condition $x_{f}$ has to be set to a value that is a bit larger that the maximum value of the selected factor (i.e. $x_{p}>x_{i}$ ). Note as well that the factors are normalized so that the model can represent anything that a user wants it to represent. Note also that the exponent is different for each factor. The exponents are selected so that the model represents some data. The only restriction is that the exponents must satisfy the initial and final conditions for each factor. The 
final condition can be an intermediate point in cases where the surface may require it. Illustrative examples are presented in the paper that shows correlation with measured data and different applications.

In this investigation, PBS is assumed to be described by the MFIM shown in the following equation:

$$
\frac{W}{W_{0}}=\left[1-\frac{V D}{V D_{f}}\right]^{-0.282}\left[1-\frac{V H}{V H_{f}}\right]^{-0.131}\left[1-\frac{F H}{F H_{f}}\right]^{-1.189}
$$

Table 1: $\quad$ Probabilistic results compared with test data from cryo ingestion tests.

\begin{tabular}{|c|c|c|c|c|c|c|c|c|}
\hline \multicolumn{2}{|c|}{$\begin{array}{c}\text { Void diameter } \\
\text { VD, } \\
\text { in. }\end{array}$} & \multicolumn{2}{c|}{$\begin{array}{c}\text { Void depth VH, } \\
\text { in. }\end{array}$} & \multicolumn{2}{c|}{$\begin{array}{c}\text { Foam over void } \\
\text { FH, } \\
\text { in. }\end{array}$} & $\begin{array}{c}\text { Test } \\
\text { divot } \\
\text { weight, } \\
\text { lb }\end{array}$ & $\begin{array}{c}\text { MFIM } \\
\text { divot } \\
\text { weight, } \\
\text { lb }\end{array}$ & $\begin{array}{c}\text { Actual } \\
\text { difference, } \\
\text { (Test- } \\
\text { MFIM), } \\
\text { lb }\end{array}$ \\
\hline MFIM & Test & MFIM & Test & MFIM & Test & & & \\
1.1249 & 1.1250 & 0.5000 & 0.5000 & 0.5000 & 0.5000 & 0.0019 & 0.0059 & -0.0040 \\
1.6248 & 1.6250 & 0.5000 & 0.5000 & 0.5000 & 0.5000 & 0.0034 & 0.0073 & -0.0039 \\
0.8749 & 0.8750 & 0.2500 & 0.2500 & 0.7499 & 0.7500 & 0.0039 & 0.0052 & -0.0013 \\
1.1249 & 1.1250 & 0.5000 & 0.5000 & 0.9999 & 1.0000 & 0.0081 & 0.0061 & 0.0020 \\
1.3749 & 1.3750 & 0.7499 & 0.7500 & 0.7499 & 0.7500 & 0.0051 & 0.0072 & -0.0021 \\
1.8748 & 1.8750 & 0.7499 & 0.7500 & 0.7499 & 0.7500 & 0.0055 & 0.0099 & -0.0044 \\
0.8749 & 0.8750 & 0.2500 & 0.2500 & 1.2499 & 1.2500 & 0.0072 & 0.0054 & 0.0018 \\
2.1229 & 2.1250 & 0.1250 & 0.1250 & 2.4998 & 2.5000 & 0.0810 & 0.0833 & -0.0023 \\
2.1235 & 2.1250 & 0.6249 & 0.6250 & 1.9998 & 2.0000 & 0.0471 & 0.0488 & -0.0017 \\
2.1191 & 2.1250 & 1.1249 & 1.1250 & 1.4999 & 1.5000 & 0.0272 & 0.0330 & -0.0058 \\
1.8748 & 1.8750 & 1.6450 & 1.7500 & 1.2499 & 1.2500 & 0.0172 & 0.0271 & -0.0099 \\
1.3749 & 1.3750 & 1.6450 & 1.7500 & 1.2499 & 1.2500 & 0.0221 & 0.0196 & 0.0025 \\
1.1249 & 1.1250 & 1.4999 & 1.5000 & 1.4999 & 1.5000 & 0.0182 & 0.0127 & 0.0055 \\
1.1249 & 1.1250 & 0.1000 & 0.1000 & 2.0998 & 2.1000 & 0.0240 & 0.0220 & 0.0020 \\
1.3749 & 1.3750 & 0.1000 & 0.1000 & 2.0998 & 2.1000 & 0.0301 & 0.0280 & 0.0021 \\
\hline
\end{tabular}

In this solution, the exact ratio for each factor as provided in the test has been used in the MFIM model. The final condition for each factor was calculated as $120 \%$ of the maximum value that was given in the test data. The reference weight $W_{0}$ was set to 0.0060 . The results from the MFIM simulation are presented in table 1 . As shown in the table, the maximum absolute difference between the test and MFIM prediction is $0.0099 \mathrm{lb}$ and the minimum absolute difference is 0.0013 . The divot weight results obtained from the MFIM simulation are compared to the test data in figure 1 for the void diameter and for the void height in figure 2. The use of MFIM replicated the test with reasonable accuracy. The values used in this part of the probabilistic evaluation are given in table 2. The probabilistic vectors for design $1 / 10,000$ and 9999/10,000 are given in table 3. The cumulative distribution function of the divot weight is shown in figure 3 . The corresponding probability density function is shown in figure 4 . 


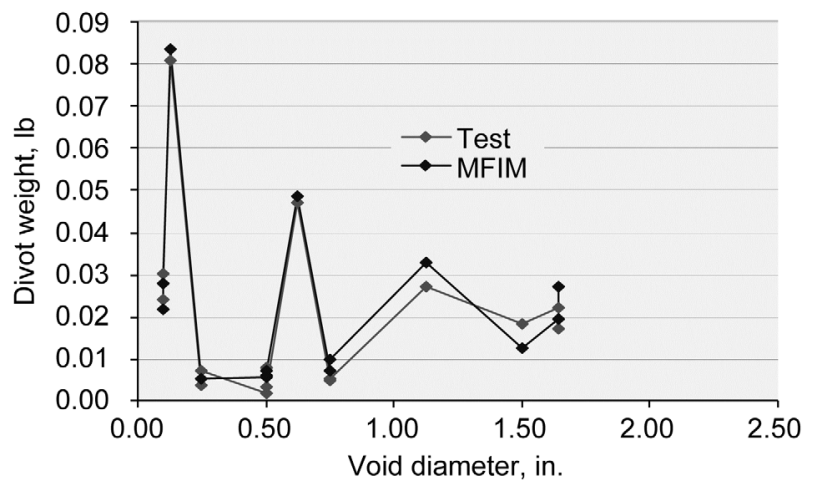

Figure 1: $\quad$ MFIM divot weight as a function of void diameter. (Cylindrical voids - cryo ingestion test).

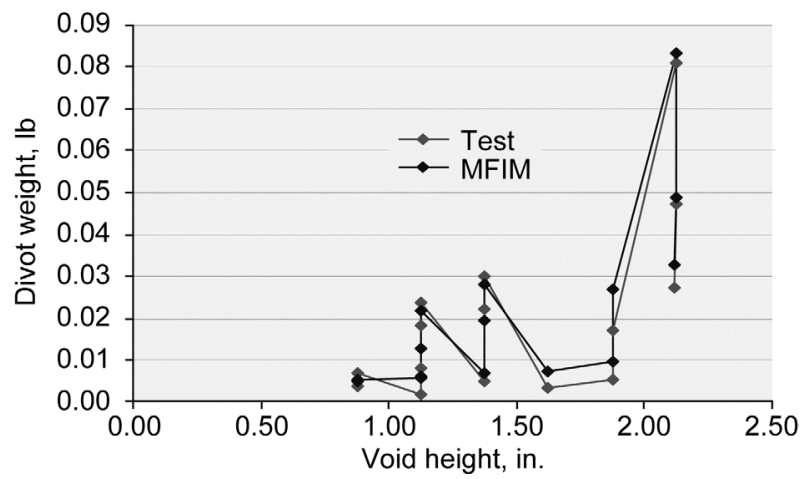

Figure 2: $\quad$ Probabilistic MFIM evaluation of divot weight (cylindrical voids_-void ingestion test).

Table 2: Variable values used in the probabilistic evaluation.

\begin{tabular}{|l|c|c|c|}
\hline \multicolumn{1}{|c|}{ Primitive variable } & Mean & $\begin{array}{c}\text { Coefficient } \\
\text { of variation }\end{array}$ & $\begin{array}{c}\text { Distribution } \\
\text { type }\end{array}$ \\
\hline Void diameter VD, in. & 1.1250 & $5 \%$ & Normal \\
Void height VH, in. & 0.5000 & $5 \%$ & Normal \\
Foam height over void FH, in. & 1.0000 & $5 \%$ & Normal \\
\hline
\end{tabular}

The respective probabilistic sensitivities are shown in figure 5. It can be seen in the summary of these results (tables and figures) that the probabilistic evaluation provides the most complete information.

The results presented in table 1 require additional discussion on how the MFIM results were obtained. Each line requires an optimization simulation as follows:

Find the values of the exponents and the corresponding vectors so that the predicted weight is close to the test weight. 
Table 3: $\quad$ Probable design vectors at $1 / 10,000$ and $9999 / 10,000$ probabilities probabilistic MFIM evaluation of divot weight (cylindrical voidscryo ingestion test).

\begin{tabular}{|l|c|c|c|}
\hline Primitive variable & Starting vector & $\begin{array}{c}0.0001 \\
\text { Probability }\end{array}$ & $\begin{array}{c}0.9999 \\
\text { Probability }\end{array}$ \\
\hline Void diameter VD, in. & 1.1250 & 0.9477 & 1.3152 \\
Void height VH, in. & 0.5000 & 0.4543 & 0.5357 \\
Foam height over void FH, in. & 1.0000 & 0.9620 & 1.0288 \\
\hline
\end{tabular}

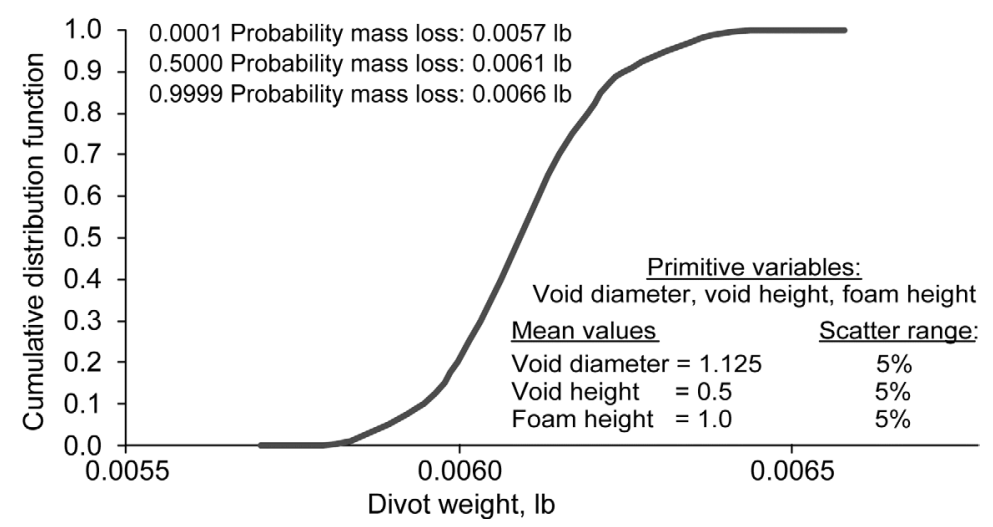

Figure 3: Preliminary MFIM probabilistic cumulative distribution function of divot weight for the cryo ingestion test (cylindrical voids).

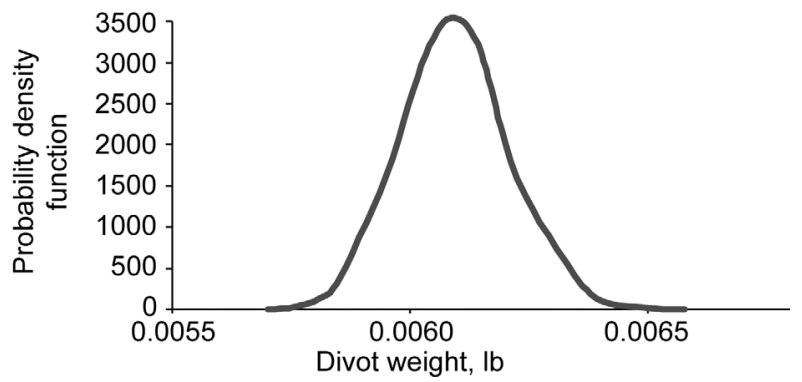

Figure 4: $\quad$ Preliminary MFIM probability density function of divot weight for the cryo ingestion test (cylindrical voids).

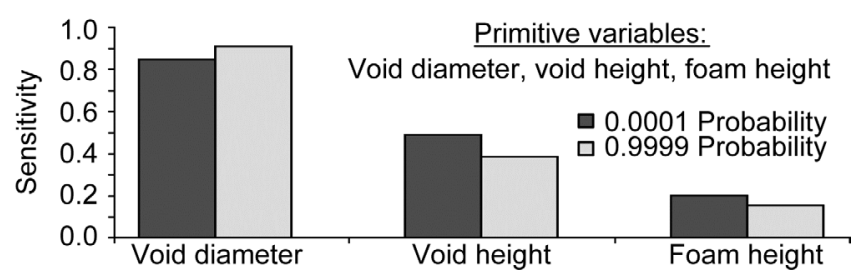

Figure 5: Preliminary MFIM probabilistic sensitivities of divot weight for the cryo ingestion test (cylindrical voids). 
Optimize $e_{x_{1}}, \ldots, e_{x_{n}} \ni W_{\text {predicted }} \approx W_{\text {test }}$ and all vectors are constrained to approximate their test values. The optimization was run as many times as there are rows in table 1 . Then the different exponents were fitted by a least squares algorithm to obtain the exponent values listed in eqn (2).

The probabilistic results are interesting. The cumulative distribution function shows the typical s-curve shape with a divot weight and almost a bell curve for the probability density function, figure 3. From the cumulative distribution function it can be seen that the divot weight is about $0.0057 \mathrm{lb}$. for a probability, figure 4 , of $1 / 1000$ and $0.0066 \mathrm{lb}$. for a probability of $9999 / 10,000$. The probability density function reads about 0.0057 to about 0.0066 .

The probability sensitivities factors are plotted in figure 5 as was already mentioned. It can be seen in this figure that the order is: void diameter, void height, and foam height above the void. The magnitudes are about 0.8, 0.5, 0.2, respectively for probability level of 0.0001 .

\section{Application of MFIM to predict foam divot in PAL ramp of the external tank}

One advantage of MFIM is that can be an effective tool where little or no information exist about a particular process or behavior. The question that would arise at this stage is what type of foam divot weight one would expect if the two variables model was applied to component specific natural voids of the External Tank (ET). The tank was dissected to determine the component specific voids in the foam. As a reminder, the foam used in the thermal protection system of the external tank is based on the application process that was in place prior to the Columbia shuttle accident. To demonstrate the effectiveness of MFIM, the reduced model shown in eqn (3) was put to use to hypothetically estimate foam divot weight based on existing voids in the PAL Ramp region of the ET. The voids from dissecting the PAL Ramp of the ET were grouped as cylindrical and slot type voids. The MFIM model of eqn (3) will address only the cylindrical voids.

$$
\frac{W}{W_{0}}=\left[1-\frac{V D}{V D_{f}}\right]^{-0.032}\left[1-\frac{V H}{V H_{f}}\right]^{-0.091}
$$

The exponents in the MFIM model were evaluated to be of $(-0.032$ and -0.091 ) based on the simulation of divot in the thermal vacuum test that was discussed earlier. The assumption here is that only two factors are present. Note that the maximum void diameter was around 0.9 in. and the maximum void height was around 0.3 in. The final condition $V D_{f}$ and $V H_{f}$ are the largest dissected void diameter and void height found in the PAL Ramp of the ET. The preliminary calculations are summarized in table 4 . The void diameter effect on the divot weight is shown in figure 6. The void height (void depth) effect on the divot weight is depicted in figure 7. MFIM, unlike any other computational model, MFIM is capable of simulating very complex behavior of functional 
responses. That is evident in the plots presented in figures 6 and 7, where the response (divot weight) took on many fluctuating trends. The analysis presented is hypothetical. The MFIM calculated divot weight requires a reference value $W_{0}$ where it can be selected, for example, as a mean value of part specific historical divot weights. In this case, it was assigned a mean value of $0.0276 \mathrm{lb}$.

Table 4: Application of MFIM to the preliminary prediction of divot weight in the PAL ramp of the external tank (cylindrical voids).

\begin{tabular}{|l|c|c|}
\hline Void diameter VD, in. & $\begin{array}{c}\text { Void height VH, } \\
\text { in. }\end{array}$ & $\begin{array}{c}\text { MFIM-divot weight } \\
W=\left(W_{0}=0.0276 \mathrm{lb}\right)\end{array}$ \\
\hline 0.2500 & 0.0500 & 0.0284 \\
0.28 & 0.1 & 0.0290 \\
0.3 & 0.2 & 0.0309 \\
0.3 & 0.03 & 0.0282 \\
0.3 & 0.1 & 0.0290 \\
0.35 & 0.05 & 0.0285 \\
0.35 & 0.15 & 0.0299 \\
0.35 & 0.05 & 0.0285 \\
0.4 & 0.05 & 0.0286 \\
0.4 & 0.1 & 0.0292 \\
0.4 & 0.02 & 0.0283 \\
0.4 & 0.1 & 0.0292 \\
0.5 & 0.1 & 0.0294 \\
0.6 & 0.29997 & 0.0659 \\
0.7 & 0.1 & 0.0300 \\
0.7 & 0.29997 & 0.0667 \\
0.89991 & 0.15 & 0.0394 \\
\hline
\end{tabular}

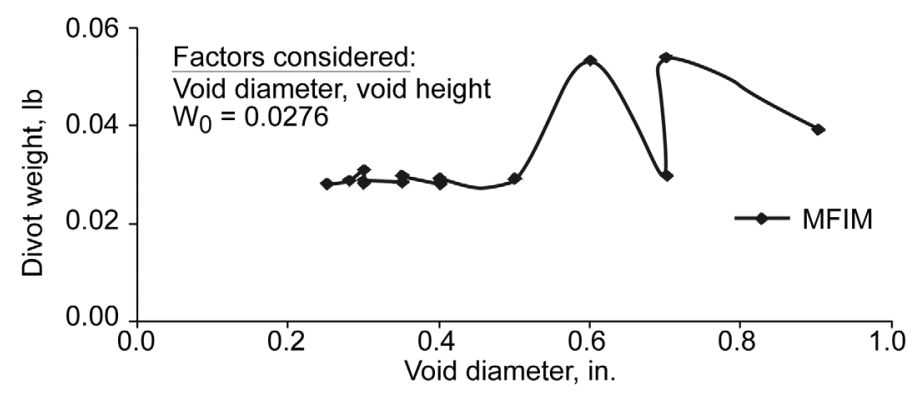

Figure 6: $\quad$ Preliminary MFIM prediction of divot weight with void diameter for the PAL Ramp of ET (cylindrical voids).

With the completion of the task of estimating the deterministic divot weight, it would be important to evaluate the probabilistic distribution and assess the influence of the foam void physical dimensions on the divot weight. The probabilistic evaluation of the divot weight for the PAL Ramp of the ET (assuming effects of thermal vacuum test) is described herein. As in the case of the deterministic model, the probabilistic MFIM model consists of the same two factors: void diameter and void height. The mean values for the void diameter and void height are, respectively, 0.434 and 0.112 . The standard deviations for the void diameter and void height are 0.11 and 0.03 in. The probabilistic 


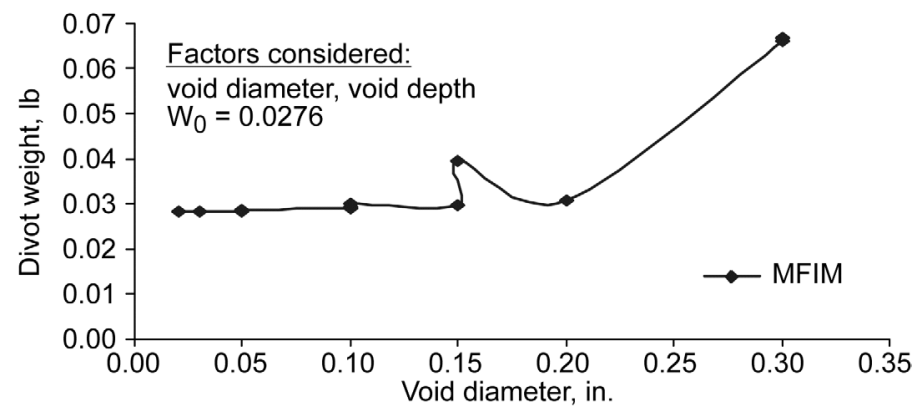

Figure 7: $\quad$ Preliminary MFIM prediction of divot weight with void diameter for the PAL Ramp of ET (cylindrical voids).

distribution type for the two independent variables, void diameter and void height, is assumed to be Lognormal for computational convenience.

The cumulative distribution function for the divot weight is shown in figure 8 . The scatter in the divot weight is estimated to be around $0.007 \mathrm{lb}$. Based on the assumed uncertainties the divot weight is $0.0289 \mathrm{lb}$ at a cumulative probability of $1 / 10,000$ while it is $0.0296 \mathrm{lb}$ at a cumulative probability of $9999 / 10,000$. The cumulative distribution function presented in figure 8 indicates that the majority of the divots would have values close to the mean. Very few divots would have weights under $0.023 \mathrm{lb}$ and above $0.0298 \mathrm{lb}$. The Probability Density Function (PDF) of the divot weight is presented in figure 9. The PDF analysis indicates that a scatter of 7 standard deviations can be achieved for the PAL Ramp anticipated divot weight. The values of the void diameter and void height at the 1/10,000 and 9999/10,000 probabilities are tabulated in the insert in figure 8 .

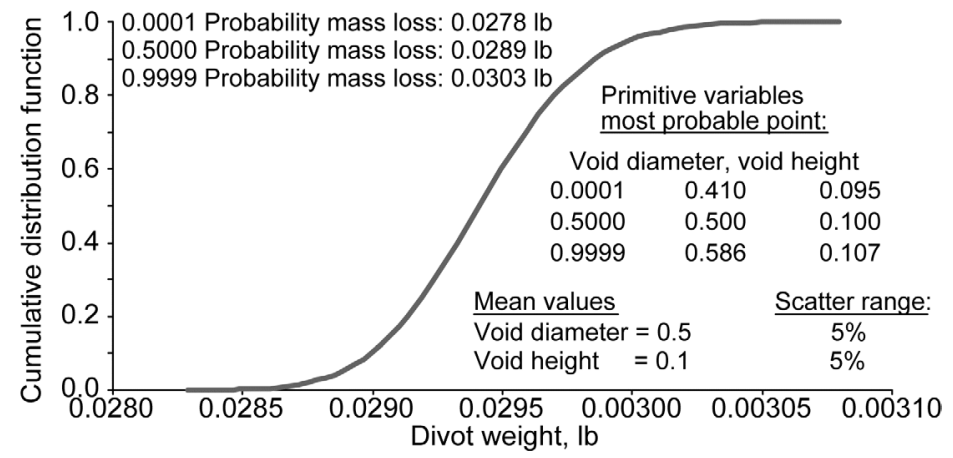

Figure 8: Preliminary MFIM probabilistic cumulative distribution function of divot weight for the PAL Ramp of ET (cylindrical voids).

An important byproduct of the probabilistic evaluation is the probabilistic sensitivities. Those are shown in figure 10. The sensitivity analysis indicates that the void diameter dominates. The void height has about $1 / 4$ of the significance in the divot weight. Unlike traditional statistical analysis, the probabilistic analysis 
can yield the design vectors that would produce a specific divot weight and also can result in calculating the design vectors that would produce near zero divot weight. Additionally, the sensitivity analysis can set the stage for eliminating from the test matrix the variables that have minimum or no effect on the divot weight. That could cut the cost and time of running additional tests using variables that would not contribute to the divot or expulsion of foam. The major conclusion from predicting computationally of divot weight is that the MFIM can be used effectively.

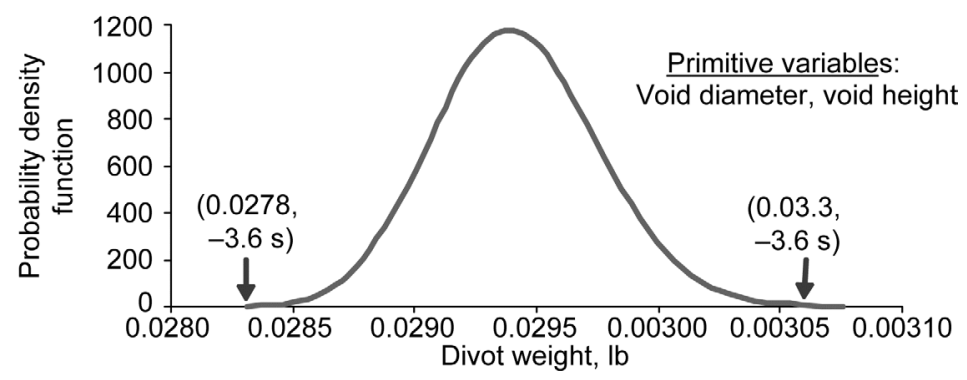

Figure 9: $\quad$ Preliminary MFIM probability density function of divot weight for the PAL Ramp ET (cylindrical voids).

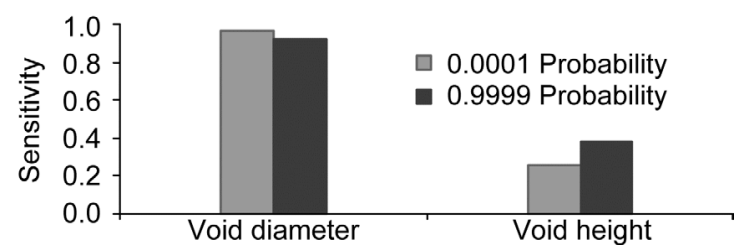

Figure 10: Preliminary MFIM probabilistic sensitivities of divot weight for the PAL Ramp of ET (cylindrical voids).

\section{MFIM with seven factors}

We now describe the effectiveness of the MFIM as applied to seven factors. Table 5 summarizes the deterministic results with the factors shown in the equation at the bottom of the table. It is noted in this table that the comparison is given on all the factors where the computed result is compared with the test result on the same line. The weight is compared in the last two columns of the table. To evaluate the probability the factors are normalized in shown in table 6 . The probabilistic values of these factors are shown in table 7 for two probabilities 0.0001 and 0.9999 . These values were obtained by asking the fast probability integrator to calculate the factors in the two probabilities. If one of the vectors was very much smaller or very much larger, then it would have been proof that these low and high probabilities were not possible and changes in the probabilities would have been required. As can be verified by visual inspection, the low and high probability values are reasonable and the probabilistic evaluation is appropriate. 
Table 5: $\quad$ Foam mass loss as predicted by multifactor interaction model (MFIM*). [Simulating mass loss in thermal vacuum test (cylindrical voids.]

\begin{tabular}{|c|c|c|c|c|c|c|c|c|c|c|c|c|c|c|c|}
\hline \multicolumn{2}{|c|}{$\begin{array}{c}\text { Void } \\
\text { diameter, } \\
V D / V D_{f}\end{array}$} & \multicolumn{2}{|c|}{$\begin{array}{l}\text { Void } \\
\text { depth, } \\
V L / V L_{f}\end{array}$} & \multicolumn{2}{|c|}{$\begin{array}{c}\text { Foam } \\
\text { thickness, } \\
F T / F T_{f}\end{array}$} & \multicolumn{2}{|c|}{$\begin{array}{l}\text { Foam } \\
\text { height, } \\
F H / F H_{f}\end{array}$} & \multicolumn{2}{|c|}{$\begin{array}{c}\text { Foam surface } \\
\text { Temperature, } \\
F S T / F S T_{f} \\
\end{array}$} & \multicolumn{2}{|c|}{$\begin{array}{c}\text { Pressure } \\
\text { inside void, } \\
P R / P R_{f}\end{array}$} & \multicolumn{2}{|c|}{$\begin{array}{c}\text { Time } \\
\text { to fail, } \\
t / t_{f}\end{array}$} & \multicolumn{2}{|c|}{$\begin{array}{c}\text { Mass loss, } \\
\mathrm{lb}, \\
M\end{array}$} \\
\hline MFIM & Test & MFIM & Test & MFIM & Test & MFIM & Test & MFIM & Test & MFIM & Test & MFIM & Test & MFIM & Test \\
\hline 0.1948 & 0.202 & 0.2085 & 0.208 & 0.4284 & 0.156 & 0.816 & 0.104 & 0.2664 & 0.237 & 0.5996 & 0.667 & 0.756 & 0.386 & 0.00040 & 0.00044 \\
\hline .1272 & .101 & .22 & .208 & .4577 & .156 & .821 & .104 & .2693 & .455 & .5625 & .556 & .825 & .495 & .00021 & .00022 \\
\hline .198 & .202 & .3889 & .417 & .6639 & .260 & .833 & .104 & .4347 & .222 & .5595 & .611 & .636 & .352 & .00040 & .00044 \\
\hline .1986 & .202 & .2106 & .208 & .6206 & .208 & .77 & .208 & .5107 & .707 & .5646 & .667 & .604 & .583 & .00132 & .00132 \\
\hline .101 & .101 & .2088 & .208 & .8295 & .208 & .235 & .208 & .2633 & .833 & .5646 & .667 & .808 & .833 & .00041 & .00044 \\
\hline .199 & .202 & .3937 & .417 & .6218 & .313 & .766 & .208 & .5088 & .671 & .5633 & .611 & .606 & .569 & .00151 & .00154 \\
\hline .1014 & .101 & .416 & .417 & .7378 & .313 & .673 & .208 & .268 & .833 & .5891 & .833 & .72 & .732 & .00041 & .00044 \\
\hline .6492 & .631 & .8226 & .833 & .35 & .833 & .55 & .833 & .6401 & .533 & .5555 & .686 & .55 & .522 & .10249 & .10318 \\
\hline .8237 & .833 & .8237 & .833 & .3508 & .833 & .553 & .833 & .6425 & .284 & .5555 & .639 & .552 & .434 & .14397 & .14506 \\
\hline
\end{tabular}

*MFIM model:

$\frac{M}{M_{0}}=\left[1-\frac{V D}{V D_{f}}\right]^{-0.5}\left[1-\frac{V H}{V H_{f}}\right]^{-0.5}\left[1-\frac{F T}{F T_{f}}\right]^{2}\left[1-\frac{F H}{F H_{f}}\right]^{2}\left[1-\frac{F S T}{F S T_{f}}\right]^{-2}\left[1-\frac{P R}{P R_{f}}\right]^{-1.5}\left[1-\frac{t}{t_{f}}\right]^{1.5}$

Table 6: Probabilistic MFIM Modeling of Foam Mass Loss. [Thermal vacuum test cylindrical voids; Mean mass loss: $0.00151 \mathrm{lb}$.

\begin{tabular}{|l|c|c|c|c|}
\hline \multicolumn{1}{|c|}{ Primitive variable } & $\begin{array}{c}\text { Normalized } \\
\text { mean }\end{array}$ & $\begin{array}{c}\text { Actual } \\
\text { mean }\end{array}$ & $\begin{array}{c}\text { Coefficient } \\
\text { of variation, } \\
\text { percent }\end{array}$ & $\begin{array}{c}\text { Distribution } \\
\text { type }\end{array}$ \\
\hline Void diameter, $V D$, in. & 0.199 & 0.99 & 5 & Normal \\
Void depth, $V H$, in. & .394 & 0.94 & 5 & Normal \\
Foam thickness, $F T$, in. & .622 & 2.98 & 5 & Normal \\
Foam height over void, $F H$, in. & .766 & 1.84 & 5 & Normal \\
Foam surface temperature, $F S T,{ }^{\circ} \mathrm{F}$ & .509 & 393.81 & 5 & Normal \\
Pressure inside void, $P R$, psi & .563 & 10.14 & 5 & Normal \\
Time to fail, $t$, sec & .606 & 89.45 & 5 & Normal \\
\hline
\end{tabular}

Table 7: Primitive variables design vectors at 0.0001 and 0.9999 probabilities.

\begin{tabular}{|l|c|c|r|}
\hline \multicolumn{1}{|c|}{ Primitive variable } & Starting vector & 0.0001 probability & $\begin{array}{r}0.9999 \\
\text { probability }\end{array}$ \\
\hline Void diameter, $V D$, in. & 0.985 & 0.984 & 0.99 \\
Void depth, $V H$, in. & .94 & .941 & .95 \\
Foam thickness, $F T$, in. & 2.98 & 3.94 & 2.25 \\
Foam height over void, $F H$, in. & 1.84 & 1.95 & 1.68 \\
Foam surface temperature, & 393.81 & 384.62 & 416.67 \\
$F S T,{ }^{\circ}$ F & & 9.92 & 10.69 \\
Pressure inside void, $P R$, psi & 10.14 & 91.93 & 84.44 \\
Time to fail, $t$, sec & 89.45 & &
\end{tabular}




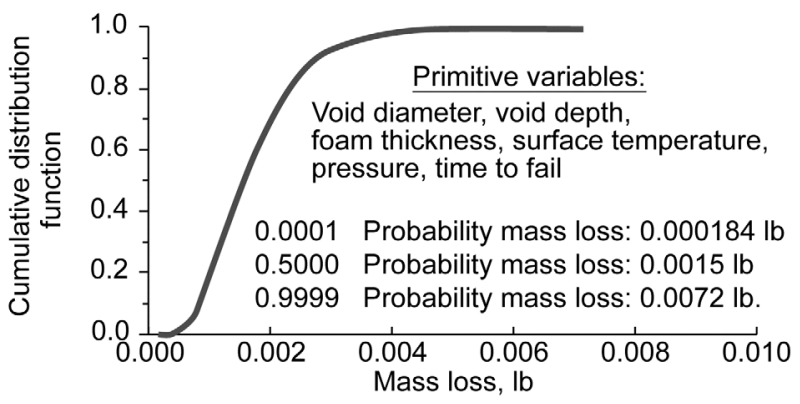

Figure 11: Probabilistic MFIM foam mass loss thermal vacuum test (cylindrical voids).

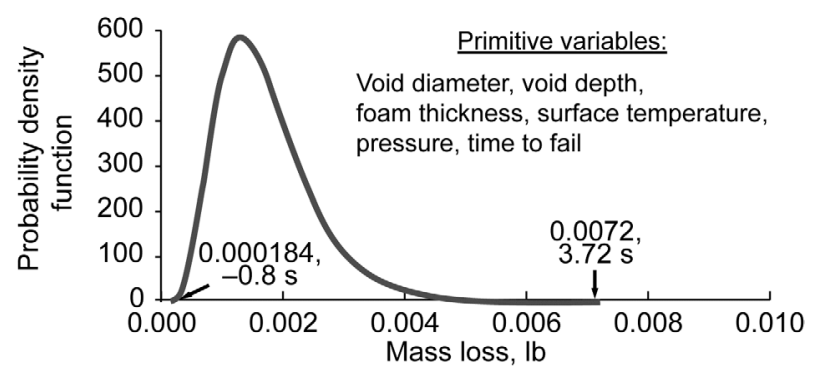

Figure 12: Probability density function with MFIM mass loss thermal vacuum test (cylindrical voids).

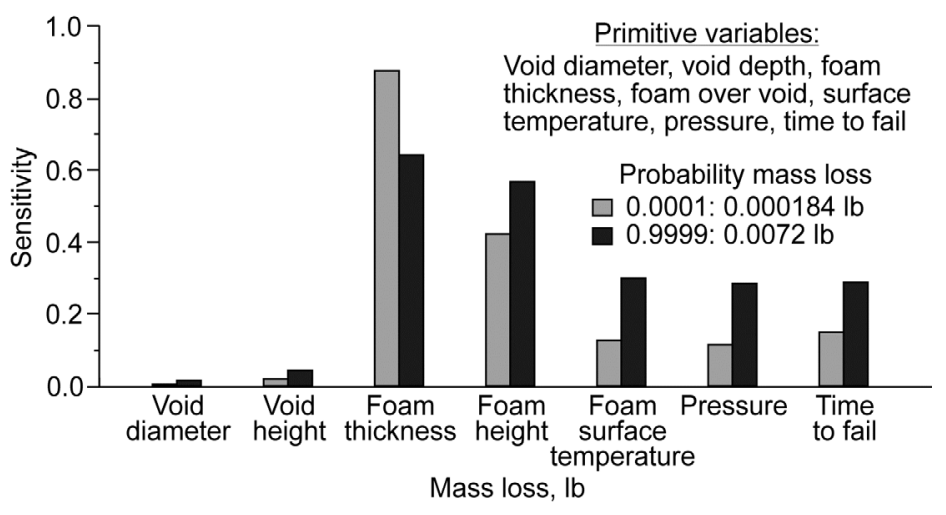

Figure 13: Probabilistic sensitivities-MFIM foam mass loss thermal vacuum test (cylindrical voids).

The CDF is plotted in figure 11. It is seen in this figure that the CDF has somewhat of an expotential distribution. This kind of a distribution is practical by the use of the fast probability algorithm. In the figure inserts the names of the factors are listed as well as three values of the CDF at probability $0.0001,0.50$, and 0.9999. As can be deduced from the respective weights in this plot, there is 
substantial weight difference. The corresponding PDF is plotted in figure 12 . Observe that the low probability value is given with respect to a standard deviation. The high probability is also given with its respective value and standard deviation. This looks like a gamma type distribution input function of the combined factors input.

The respective sensitivity factors are summarized in figure 13. It can be seen in this figure that dominant factors in a decreasing order are: foam thickness, foam height, while foam surface temperature, void internal pressure and time to fail are about of equal magnitude. The results of this last example illustrate the effectiveness of the multifactor interaction model; and even more importantly, the effectiveness of the fast probability algorithm that made the results practical with respect to computational time.

\section{Conclusions}

The Multi-Factor Interaction Model (MFIM) is a very effective way to represent factors which influence material behavior. In this investigation, MFIM was applied to predict the foam divot weight in the external tank during its ascent cycle. Factors with two, three, and seven terms were evaluated and compared with test results that were obtained from tests of simulated conditions during the ascent of launching vehicles. The comparisons were very satisfactory considering the relative small divot weight. Results also were obtained on what values the factor needs to be in order to ascertain divot weights at very low and very high probabilities. The exponents of the factors were obtained by a local optimization. The overall conclusion is that the MFIM in conjunction with the fast probability integration algorithm is very effective and practical for evaluating the MFIM and matching experimental data.

\section{References}

[1] Chamis, C.C., Lark, M.F. and Sinclair, J.H., "Integrated Theory for Predicting the Hygrothermo Mechanical Response of Advanced Composite Structural Components," ASTM STP 658, 1978, pp. 160-192.

[2] Chamis, C.C. and Hopkins, D.A., "Thermoviscoplastic Nonlinear Constitutive Relationships for Structural Analysis of High Temperature Metal Matrix Composites," NASA TM-87291. Nov. 1985.

[3] Chamis, C.C., Murthy, P.L.N. and Hopkins, D.A., "Computational Simulation of High Temperature Metal Matrix Composites Cyclic Behavior," ASTM, STP 1080, pp. 56-69.

[4] Tong, M.T., Singhal, S.N., Chamis, C.C. and Murthy, P.L.N., "Simulation of Fatigue Behavior of High Temperature Metal Matrix Composites," ASTM-Reprint from Standard Technical Publication 1253, 1996, pp. 540-551.

[5] Boyce, L. and Chamis, C.C., "Probabilistic Constitutive Relationships for Cyclic Material Strength Models," AIAA/ASME/ASCE/AHS $29^{\text {th }}$ Structures, Structural Dynamics and Materials Conference, Part 3, AIAA, 1988, pp. 1299-1306. 
[6] Minnetyan, L., "Progressive Fracture Structural Analysis of National Wind Tunnel Structures," NASA CR-198485, May 1996.

[7] Chamis, C.C. and Minnetyan, L., "A Multi-Factor Interaction Model for Damage Initiation and Progression," ASME/IMECE 2001/AD-25301, Nov. 2001 . 